\title{
Experimental and DFT Investigation into Chloride Poisoning Effects on Nitrogen-Coordinated-Iron-Carbon (FeNC) Catalysts for Oxygen Reduction Reaction
}

Deeksha Jain ${ }^{1 *}$, Qiang Zhang ${ }^{1 *}$, Vance Gustin ${ }^{1}$, Jonathan Hightower ${ }^{1}$, Seval Gunduz ${ }^{1}$, Anne C. $\mathrm{Co}^{2}$, Jeffrey T. Miller ${ }^{3}$, Aravind Asthagiri ${ }^{1 * *}$, Umit S. Ozkan $^{1 * *}$

${ }^{1}$ William G. Lowrie Department of Chemical and Biomolecular Engineering, The Ohio State University, Columbus, Ohio 43210, USA

${ }^{2}$ Department of Chemistry and Biochemistry, The Ohio State University, Columbus, Ohio 43210, USA

${ }^{3}$ Davidson School of Chemical Engineering, Purdue University, West Lafayette, Indiana 47907, USA

\section{Supplementary Information}

*Deeksha Jain and Qiang Zhang contributed equally to this work.

**Corresponding authors

ozkan.1@,osu.edu, +1-614-292-6623

asthagiri.1@,osu.edu, +1-614-688-8882 


\section{1s}

\begin{tabular}{|c|c|c|c|c|}
\hline Sample & C & N & O & Cl \\
\hline FeNC & 98 & 1 & 1 & 0 \\
\hline FeNC-Cl & 96 & 1 & 2 & 1 \\
\hline
\end{tabular}

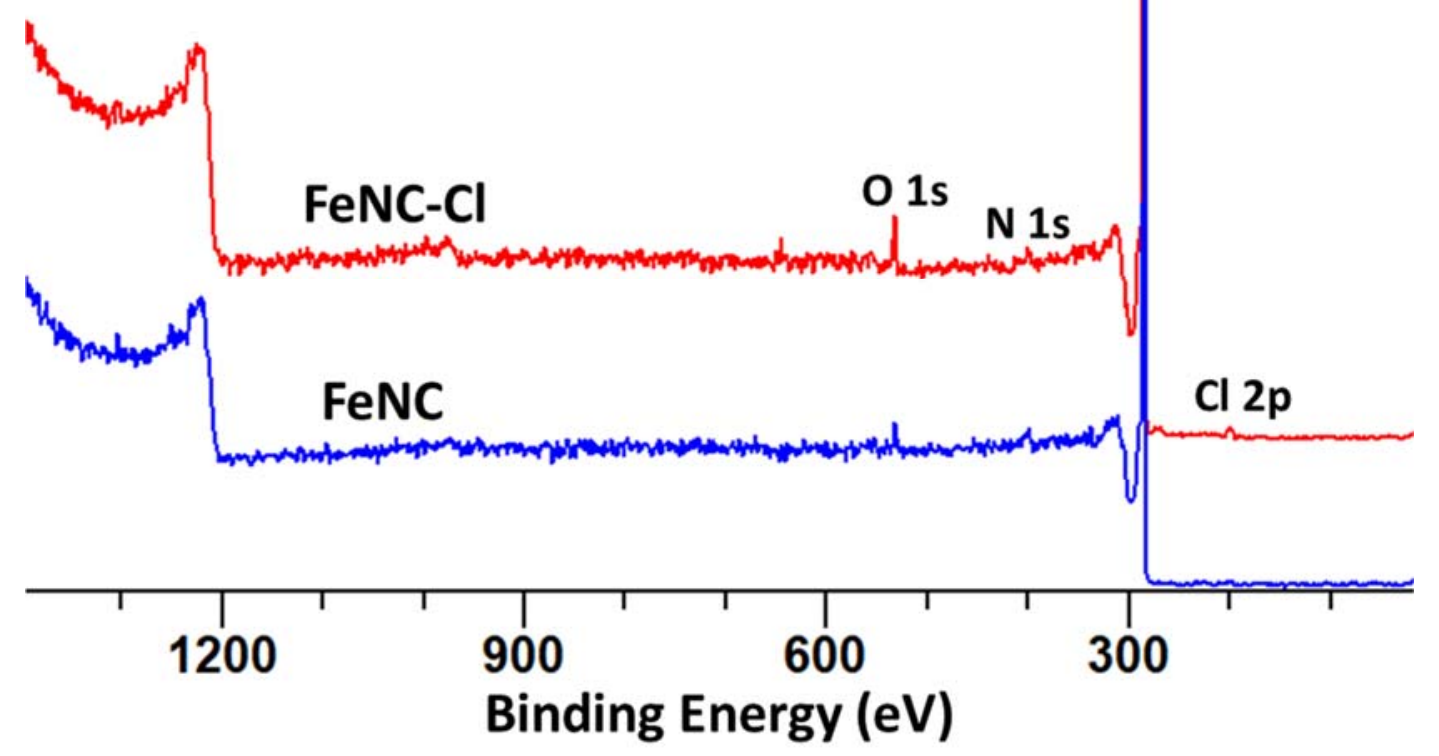

Figure S1: XPS survey spectra of FeNC and FeNC-Cl samples. Inset: Surface atomic composition (\%) obtained for FeNC and FeNC-Cl samples 


\section{(a) FeNC (0.5 $\left.\mathrm{M} \mathrm{H}_{2} \mathrm{SO}_{4}\right)$}

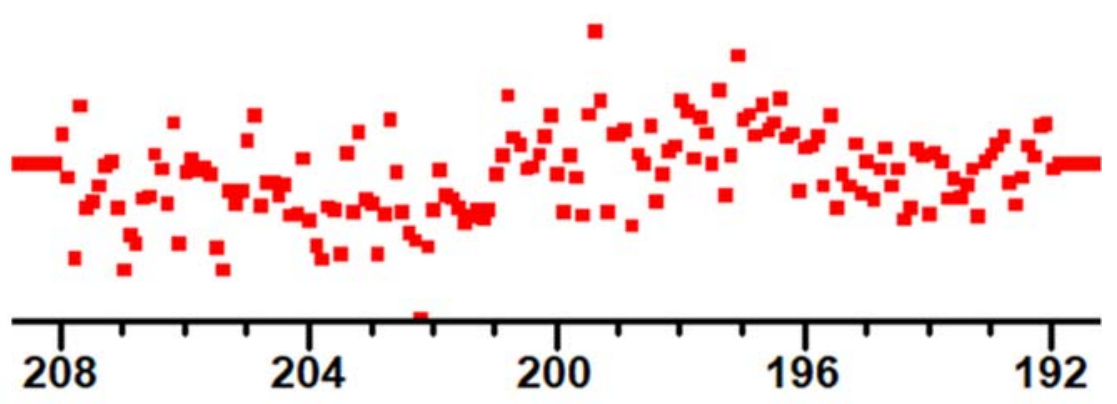

\section{(b) $\mathrm{FeNC}\left(0.5 \mathrm{M} \mathrm{H}_{2} \mathrm{SO}_{4}+1 \mathrm{M} \mathrm{NaCl}\right)$}

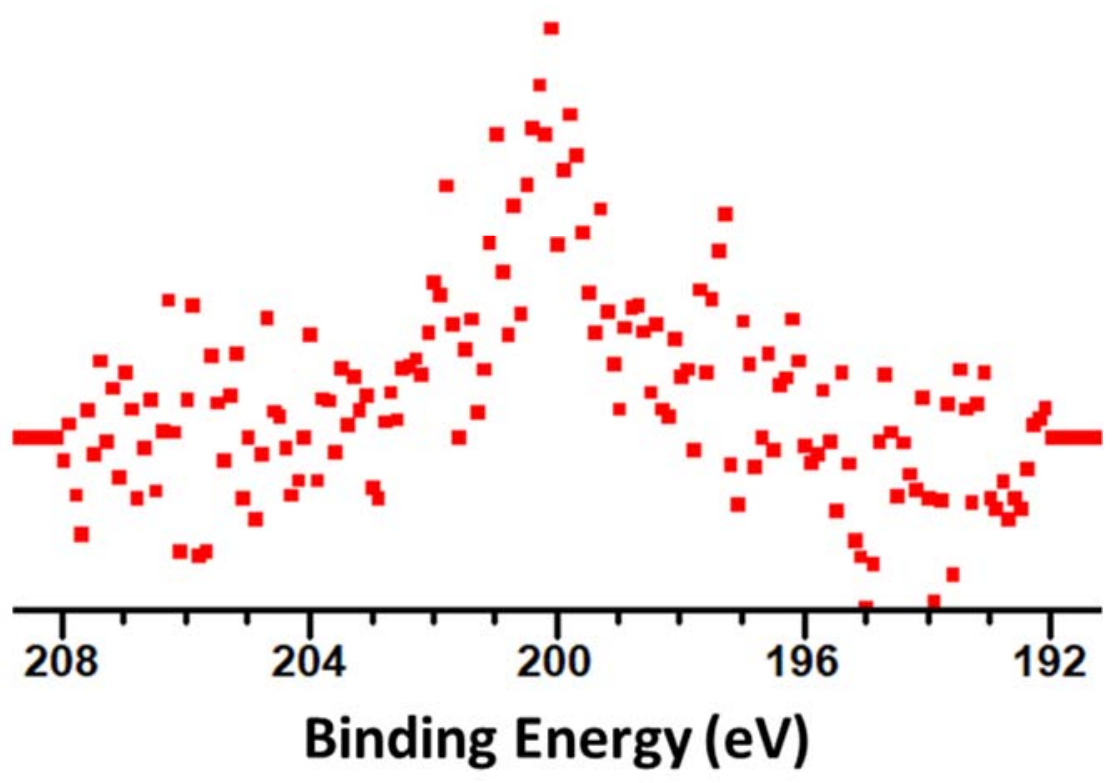

Figure S2: Post reaction XPS spectra in the Cl-2p region of the FeNC sample before and after chloride exposure in argon-saturated electrolyte, with electrode potential held constant at $1 \mathrm{~V}$ vs. RHE 

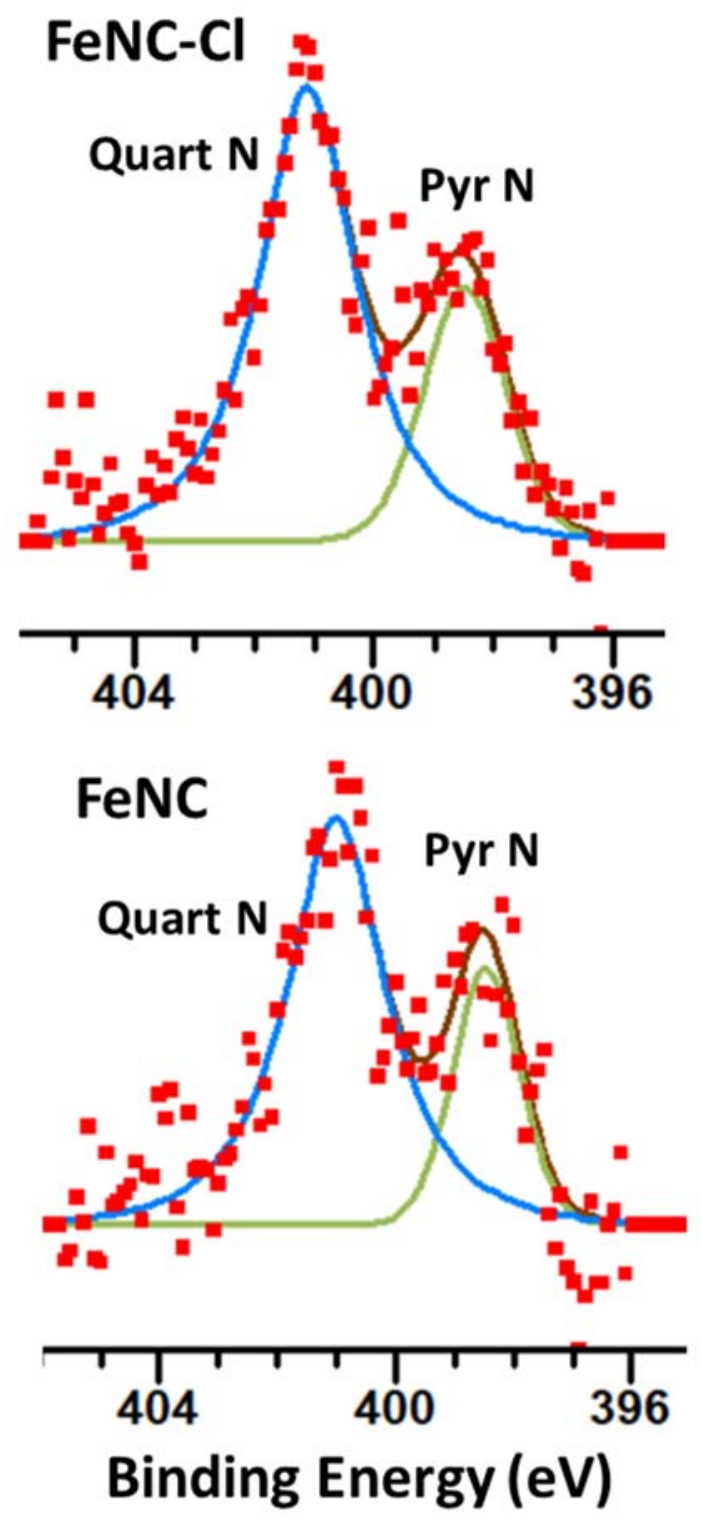

Figure S3: XPS spectra of FeNC and $\mathrm{FeNC}-\mathrm{Cl}$ samples obtained in the $\mathrm{N}$ 1s region 


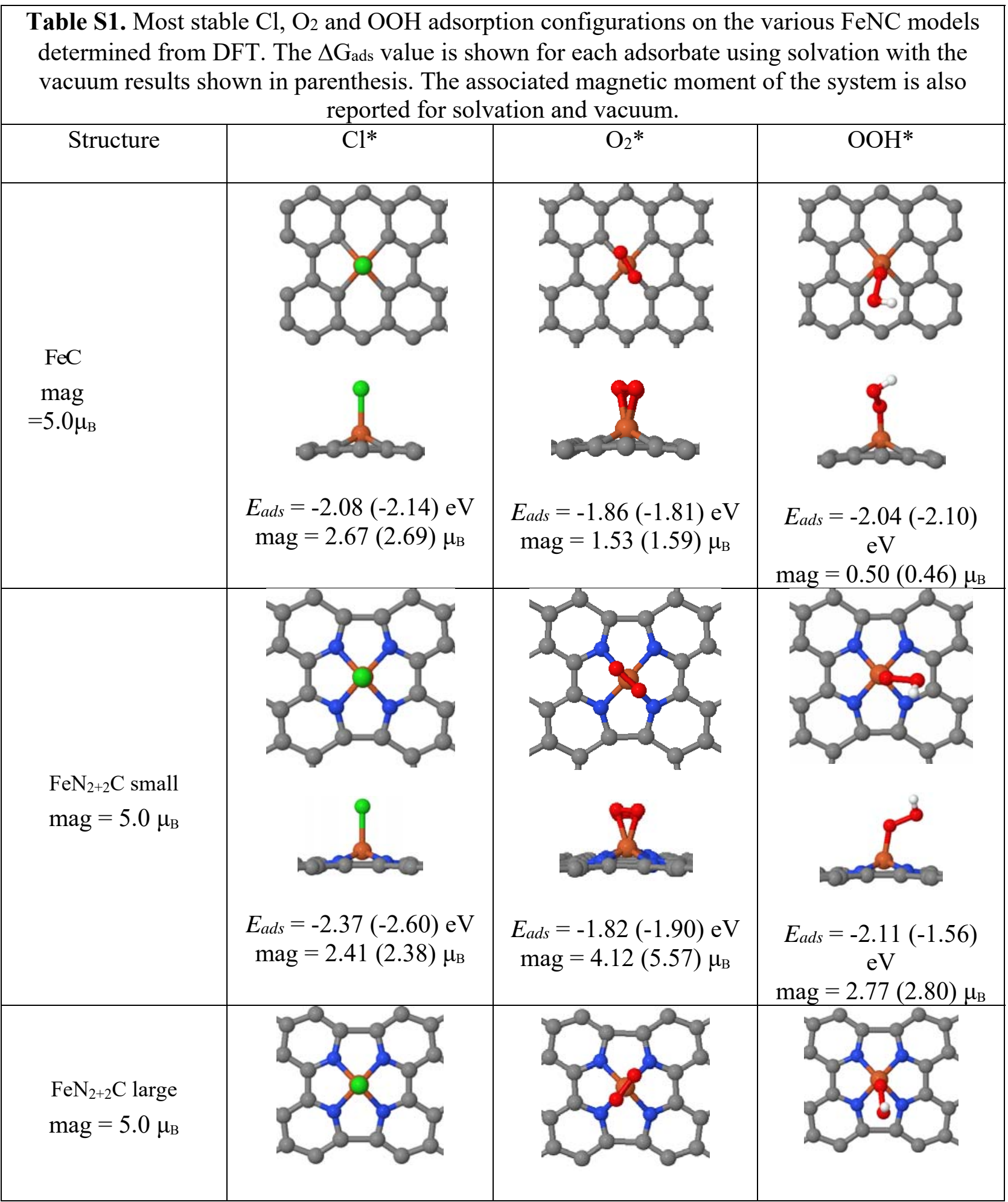




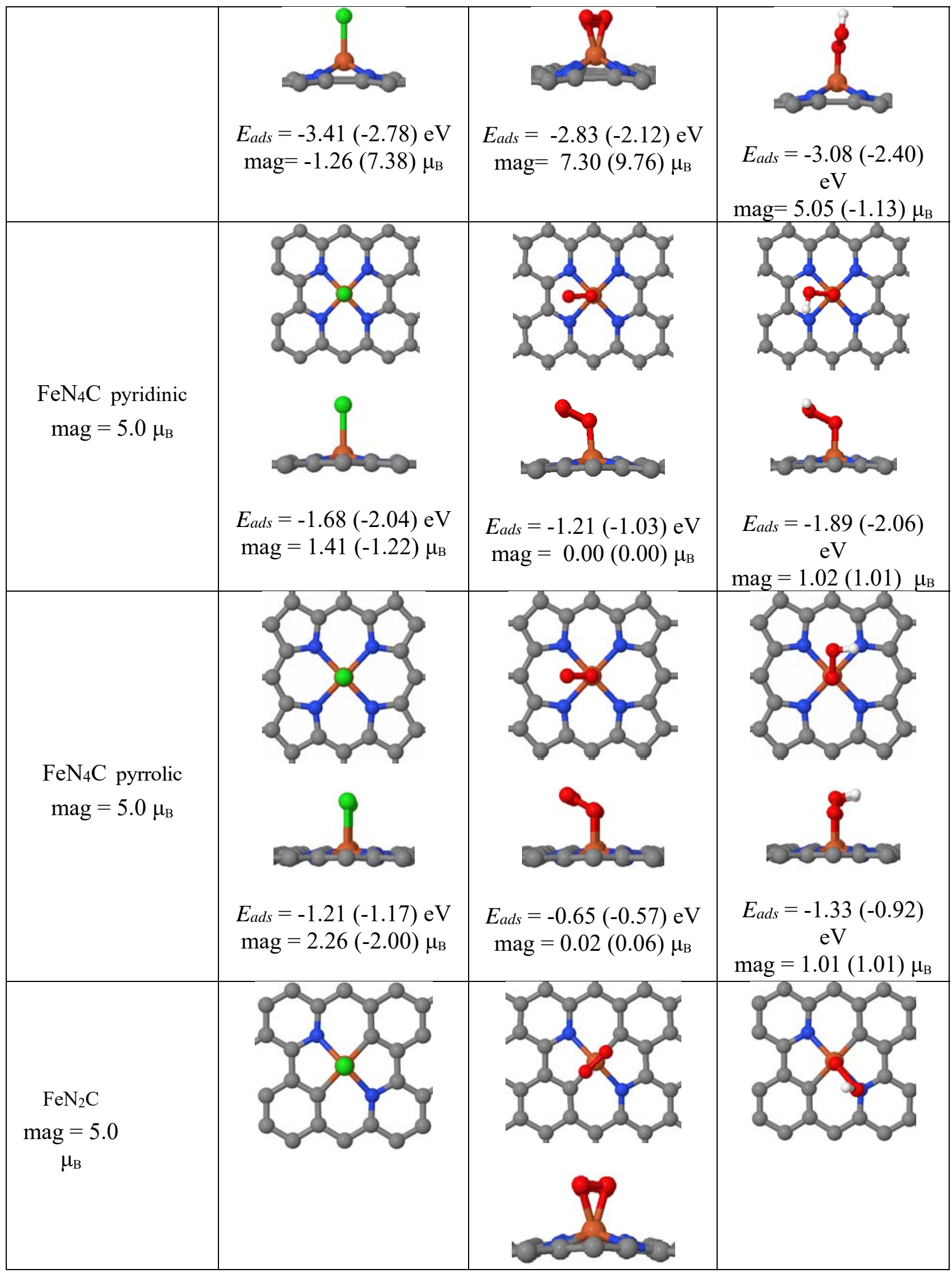




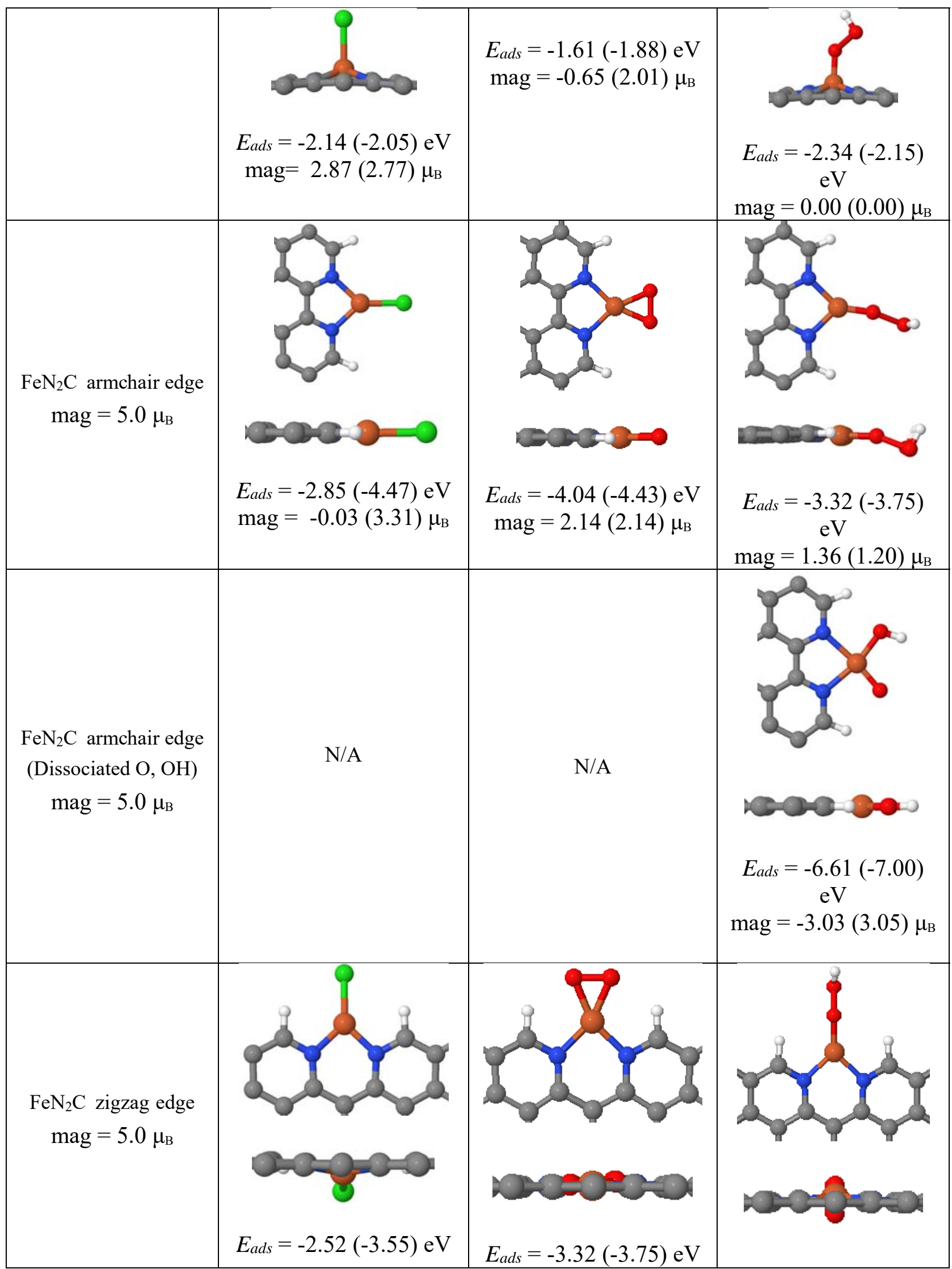




\begin{tabular}{|c|c|c|c|}
\hline & $\operatorname{mag}=-0.77(0.41) \mu_{\mathrm{B}}$ & $\operatorname{mag}=1.36(3.00) \mu_{\mathrm{B}}$ & $\begin{array}{c}\text { Eads }=-2.58(-3.08) \\
\mathrm{eV} \\
\operatorname{mag}=-2.18(-2.27) \\
\mu_{\mathrm{B}} \\
\end{array}$ \\
\hline $\begin{array}{c}\mathrm{FeN}_{2} \mathrm{C} \text { zigzag edge } \\
\text { (Dissociated } \mathrm{O}, \\
\mathrm{OH}) \\
\operatorname{mag}=5.0 \mu_{\mathrm{B}}\end{array}$ & N/A & N/A & $\begin{array}{c}E_{a d s}=-5.67(-6.05) \\
\mathrm{eV} \\
\mathrm{mag}=2.20(-2.30) \mu_{\mathrm{B}}\end{array}$ \\
\hline
\end{tabular}




\begin{tabular}{|c|c|c|c|}
\hline Model & Bare surface & $\begin{array}{c}\text { Cl adsorbed } \\
\text { surface } \\
\end{array}$ & $\begin{array}{l}\text { Core level binding } \\
\text { energy shifts (eV) }\end{array}$ \\
\hline $\mathrm{FeN}_{2} \mathrm{C}$ armchair & 400.0 & 400.3 & 0.3 \\
\hline $\mathrm{FeN}_{2} \mathrm{C}$ zigzag & 400.5 & 400.7 & 0.2 \\
\hline $\mathrm{FeN}_{4} \mathrm{C}$ small pore & 400.5 & 400.8 & 0.3 \\
\hline $\mathrm{FeN}_{4} \mathrm{C}$ large pore & 400.5 & 400.9 & 0.4 \\
\hline $\mathrm{FeN}_{4} \mathrm{C}$ pyridinic & 399.9 & 401.2 & 1.3 \\
\hline $\mathrm{FeN}_{2} \mathrm{C}$ & 400.1 & 401.2 & 1.0 \\
\hline $\mathrm{FeN}_{4} \mathrm{C}$ pyrrolic & 400.0 & 401.1 & 1.1 \\
\hline
\end{tabular}

\begin{tabular}{|c|c|c|}
\hline \multicolumn{2}{|c|}{$\begin{array}{c}\text { Table S3: Experimentally determined relative distribution of } \\
\text { various species in N-1s region of XPS }\end{array}$} \\
\hline Sample & $\begin{array}{c}\text { Pyridinic N } \\
\mathbf{( 3 9 8 . 5} \mathbf{~ e V )}\end{array}$ & $\begin{array}{c}\text { Quaternary N } \\
(\mathbf{4 0 1} \mathbf{~ e V )}\end{array}$ \\
\hline FeNC & 27 & 73 \\
\hline FeNC-Cl & 29 & 71 \\
\hline
\end{tabular}

